\title{
A Proposed Algorithm to Detect the Effect of an Object Within a Video Series
}

\author{
Abeer Abd Al-Khaliq Thanoon \\ College of Law
}

University of Mosul

ReceivingOn: 10/6/2009

AcceptanceOn: 21/2/2010

\begin{abstract}
In this research an algorithm for detecting a specific object and tracing its movement within a depicted series is proposed. A video file of the AVI type has been used since this type comprises a group of depicted frames. The dynamic path of the required target detected has been traced. It takes the form of a walking person. At the beginning, the detecting stage involves reading the frame and analyzing the structure of the picture by using co-occurrence matrix. The features of the picture's structure are then represented in the form of a chart, followed by cutting up the picture depending on the values of the chart in distributing of colours in order to get the required detected object to be traced. The location of the object is then specified through the calculate of the area center of the target in each frame , and after that drawing its dynamic path by using Euclidean Law for area along the depicted series.

Keywords: Algorithms, video file of the AVI type.
\end{abstract}

$$
\begin{aligned}
& \text { خوارزمية مقترحة لكثف آثر جسم ضمن سلسلة فيديوية } \\
& \text { عبير عبد الخالق ذنون } \\
& \text { كلية الحقوق } \\
& \text { جامعة الموصل }
\end{aligned}
$$

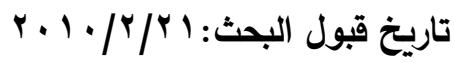

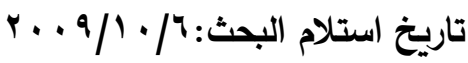


الفيديوية ، لقد تم تعقب المسار الحركي لنوع الجسم المراد اكتثافه وهو شخص يمشي .في البداية تكون مرحلة الاكتثاف وذلك من خلال قراءة الإطار ثم تحليل نسيج الصورة باستخدام المصفوفة الظاهرة ثم تمثيل

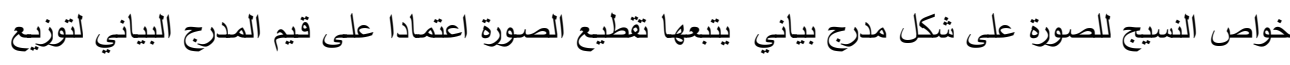

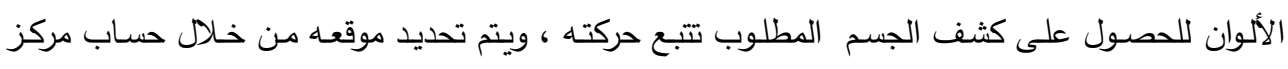
المنطقة للهدف في كل إطار ورسم المسار الحركي لله باستخدام قانون إقليدس للمسافة على طول السئل السلسلة

AVI الكلمات : الخوارزميات ، ملف فيوي من نوع

1-1

هنالك العديد من التقنيات المستخدمة لتقطيع الصور الرقمية وإن اختيار الطريقة المناسبة

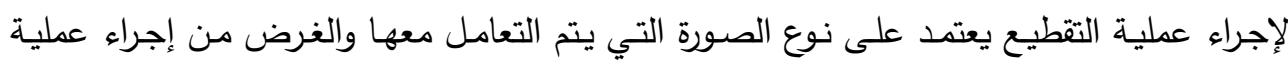

التقطيع ، وحسب الحاجة لتلك التقنية وملائمتها للعمل.

من التقنيات المستخدمة في هذا المجال تقنية تحليل نسيج الصورة ، حيث تعد هذه الطريقة

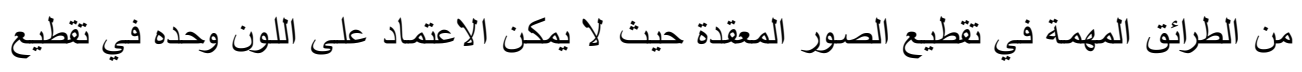

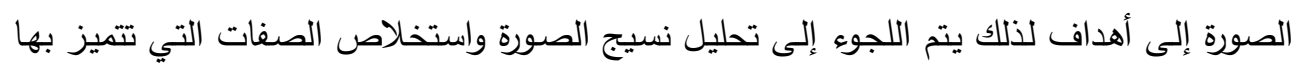

إن عملية كثف الأجسام في سلسلة فيديوية يمكن التعريف عنها بالثكل التالي (ظهور

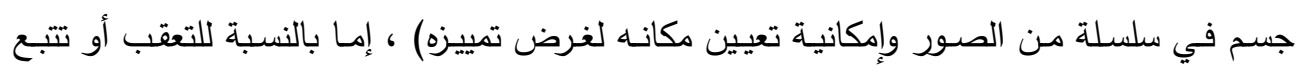

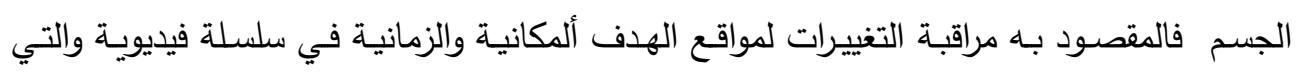

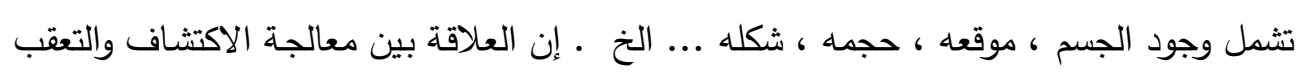

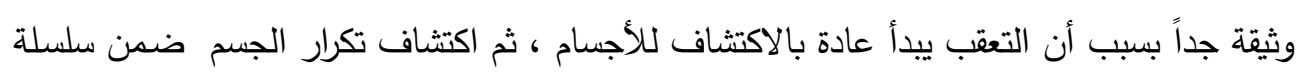

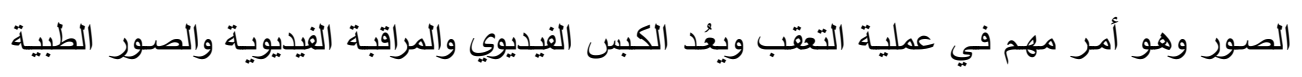
والانسان الألي ... الخ من تطبيقات الكثف وتتبع الأهداف في الرؤيا الحاسوبية [3].

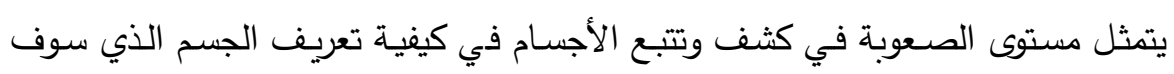

يُكثف والذي يتم تعقبه بعد ذلك ضمن السلسلة الصورية وتوجد عدة طرائق للكثف عن الجسم منها

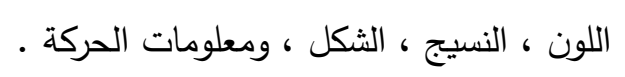

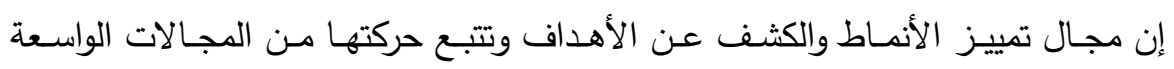

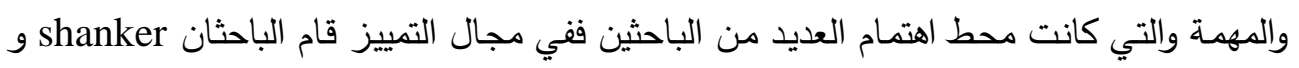

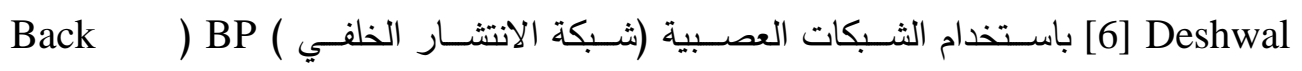

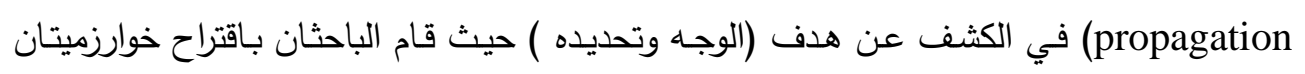


للتمييـز الأولـى باسـتخدام شـبكة BP والأخــرى باسـتخدام خوارزميــة المكنـــة المتجهـة SVM وإجراء المقارنة بينهما. وكذلك في مجال الكثف عن الجسم قام

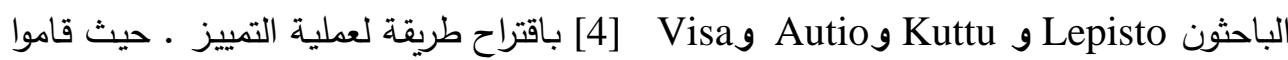
بأخذ عينة لغرض عملية التمييز ومن ثم التصنيف ـو ـوتم تصنيف نسيج الحصى بالاعتماد فيها

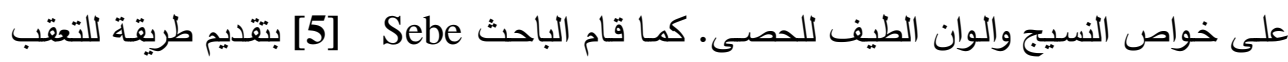
اعتمادأ على ثلاث محددات اللون والحافة والحركة ووضح فيها ضرورة اعتماد أكثر من محدد لتعقب

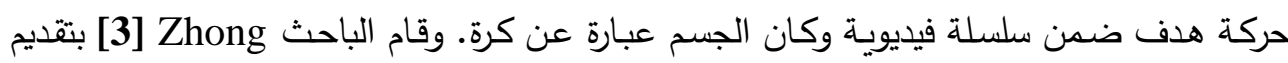
دراسة عن الطرق والبحوث التي عملت في مجال الكثف وعملت في مجال التعقب وكلاهما معاً

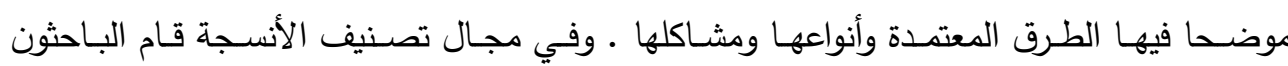
[8 Philips, Li, Jacob, Daniela

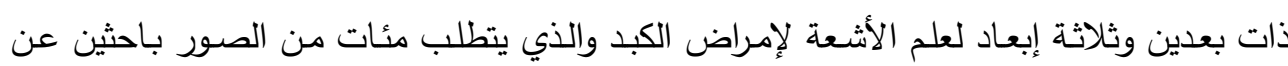

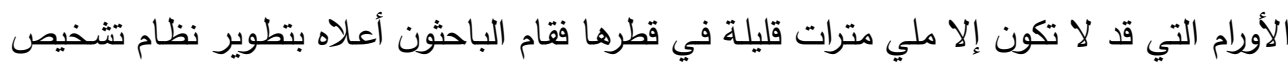
باستخدام الحاسوب من خلال إجراء مقارنة بين خوارزميتين ثلاثية الأبعاد ونظيرتها ثنائية الأبعاد

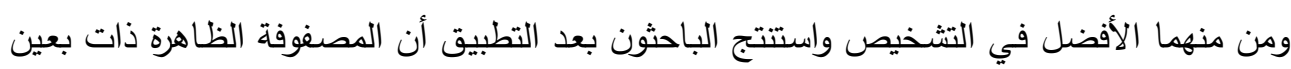

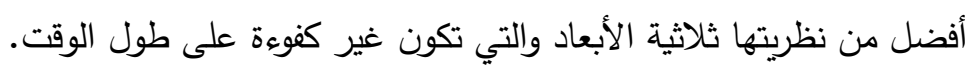

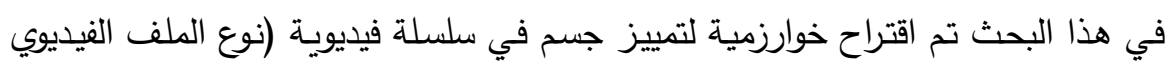

(AVI CO-Occurrence Matrix

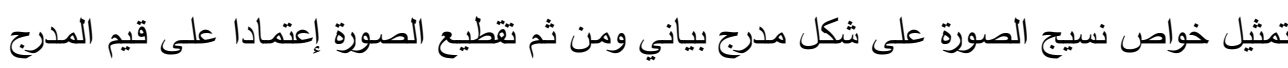

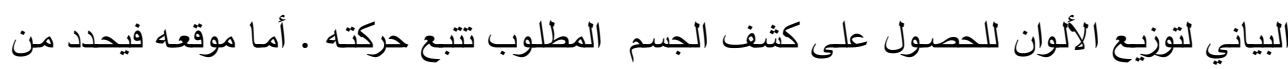
خـلال حساب مركز المنطقة في كل إطـار ثم رسم المسار الحركي لله باستخدام قانون المسـافة . Euclidean distance السلسلة الصورية للملف الصوري .

\section{2- الخوارذمية المقترحة لكثف الجسم والتتبع:}

$$
\text { تتضمن الخوارزمية الخطوات التالية : }
$$

1- تحليل نسيج الصورة باستخدام المصفوفة الظاهرة Co-Occurrence Matrix وهي أحسات

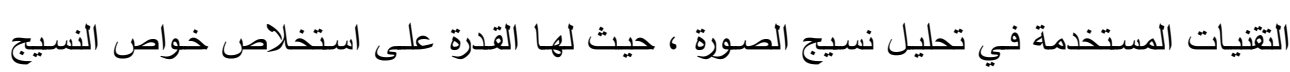
لمختلف أنواع الصور الرقمية كما ويستخدم هذا التحليل في عملية تصنيف الصور عند معالجة 
الصور الرمادية ويتم الحصول على المصفوفة الظاهرة بعد إجراء عملية تعيير (Normalization) وكما في المعادلات التالية [2] : $\mathrm{r}=\frac{R}{R^{*} G^{*} B}$

$\mathrm{g}=\frac{G}{R^{*} G^{*} B}$

$\mathrm{b}=\frac{B}{R^{*} G^{*} B}$

إن المصفوفة الظاهرة تعتمد على تكرار الظهور لترتيب عدد من المستويات الرمادية في الأنسجة تشكل هذه التكرارات ، وإن المصفوفة الظـاهرة هي مصفوفة مربعة بالاعتمـاد على تردد الظهور

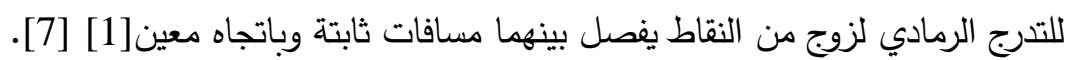
$\mathrm{Co}=\sum_{i, j} \frac{p(i, j)}{1+|i-j|}$

$$
\text { حيث أن }
$$

Do

p

i

j : إحداثيات العمود

i,j

p(i,j)

ويتم تمثيل خواص نسيج الصورة على شكل مدرج بياني Histogram وكما في الثكل (1).

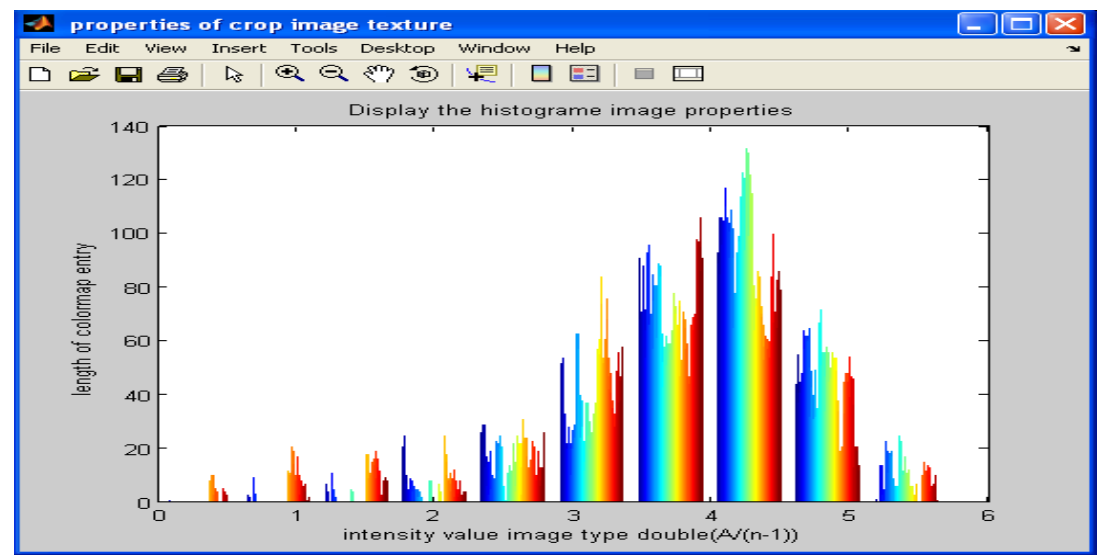




$$
\text { شكل (1) المدرج البياني لتمثيل خواص الصورة }
$$

2- تقطيع الصور باستخدام حد العتبة في التدرج البياني حيث تعد عملية تقطيع الصور من أهم

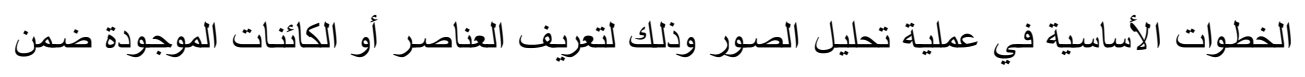

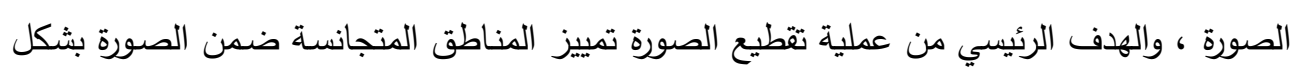

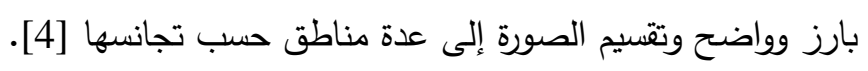

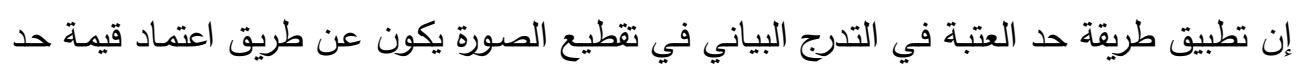

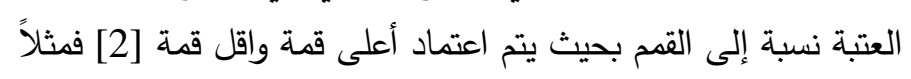

$\mathrm{T} \geq 2$

حيث أن T T Tيمة العتبة الناتجة عن النسبة بين أعلى قمة واقل قمة في المدرج التكراري .

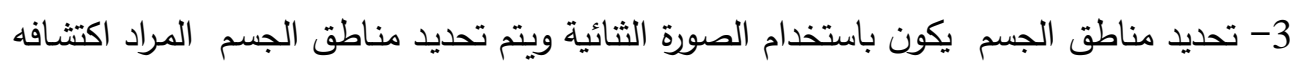

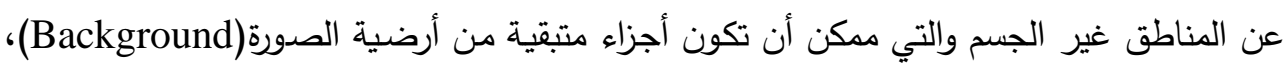

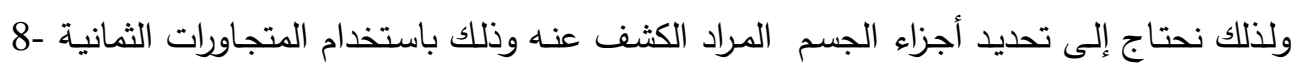
connected neighborhood لكل أجزاء الجسم (الوحدات الصورية تكوّن متجاورات مترابطة

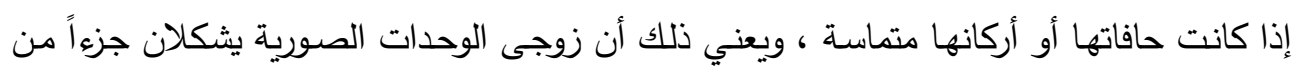

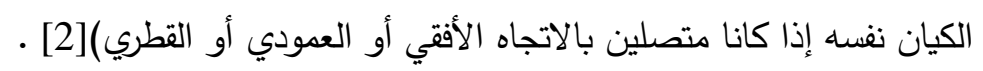

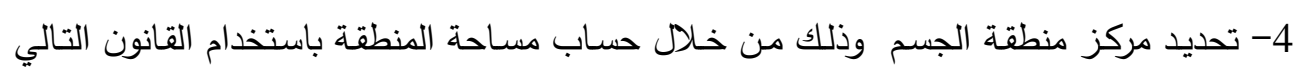

$\mathrm{Xx}=1 / \mathrm{A} \sum \sum \mathrm{j} \mathrm{B}_{(\mathrm{i}, \mathrm{j})}$

$\mathrm{Yy}=1 / \mathrm{A} \sum \sum \mathrm{i} \mathrm{B}_{(\mathrm{i}, \mathrm{j})}$

: A

[n*m [مثل المنطقة . مصفوفة ذات بعدين : B

$\mathrm{Xc}=$ obj Center(:,1);

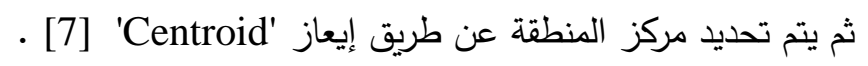

Yc $=$ obj Center(:,2);

5- تتبع الجسم من خلال حساب المسار الحركي له على طول السلسلة الصورية وذلك من خلال

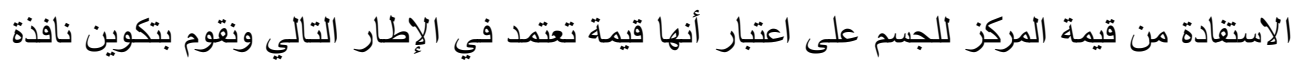


محيطة بالجسم اعتمادا على قيمة المركز الناتجة نصف طول هذه النافذة 40 نقطة صورية وحسب المعادلتين التاليتين.

New $X=X c+$ WindowCenter

New $\mathrm{Y}=\mathrm{Yc}+$ WindowCenter

وباستخدام قانون المسافة يتم حساب طول المسار الحركي للهدف بعد حساب المركز في كل إطار

Distance $=\sqrt{\left(x_{1}-x_{2}\right)^{2}+\left(y_{1}-y_{2}\right)^{2}}$

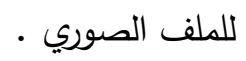

$$
\begin{aligned}
& \text { : قيمة المركز للاحداثي السيني للإطار الأول X1 } \\
& \text { قيمة المركز للاحداثي السيني للأطار الثاني : X2 }
\end{aligned}
$$

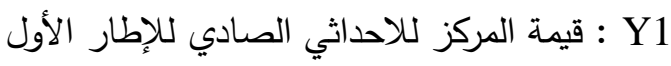

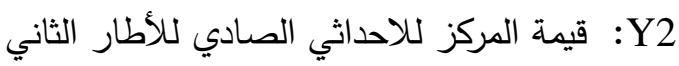

$$
\begin{aligned}
& \text { 3- الجانب العملي والتطبقي للخوارنمية المقترحة }
\end{aligned}
$$

تم استخدام لغة Matlab8.0 لغرض بناء وتصميم برنامج نظام مراقبة للمثالين التاليين:

$$
\text { 1- المثال التطبيقي الأول وحسب الخطوات التالية :- }
$$

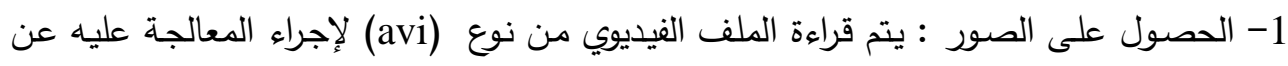

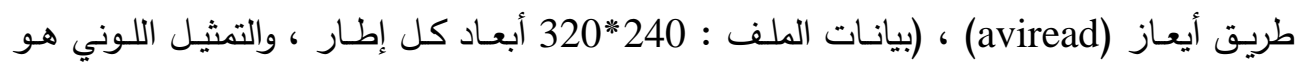

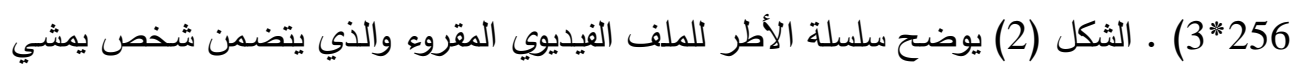
، اما الثكل (3) فيوضح عرض سلسلة الأطر لكن بطريقة أخرى.

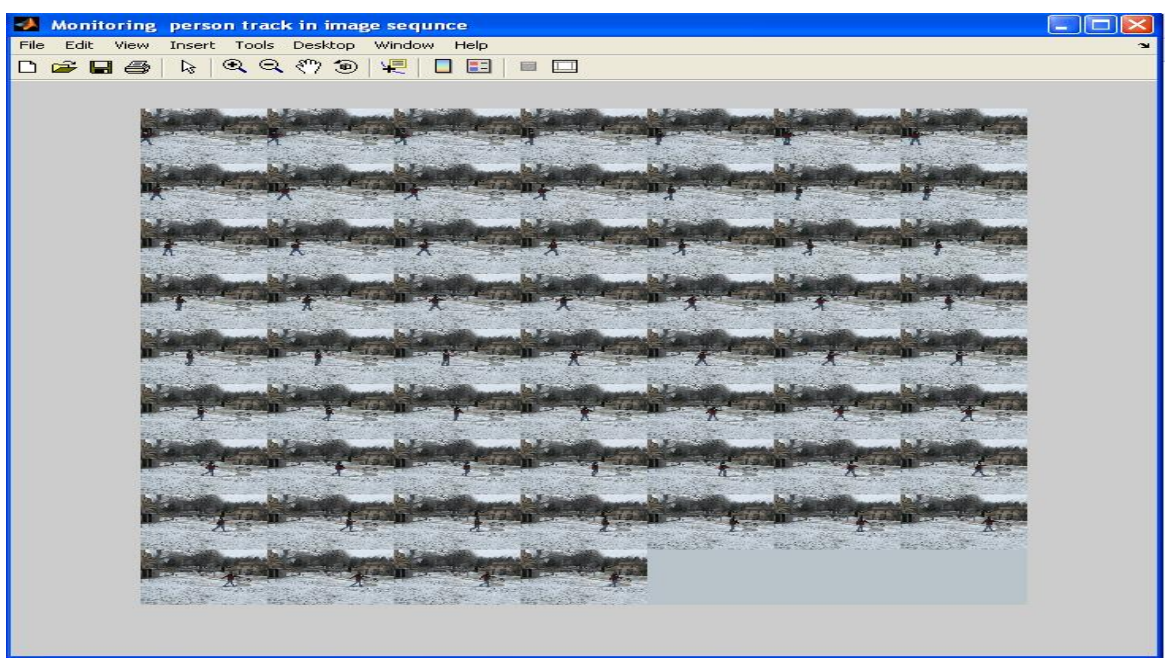




\section{شكل (2) عرض الأطر المقروءة ضمن الملف الفيديوي}

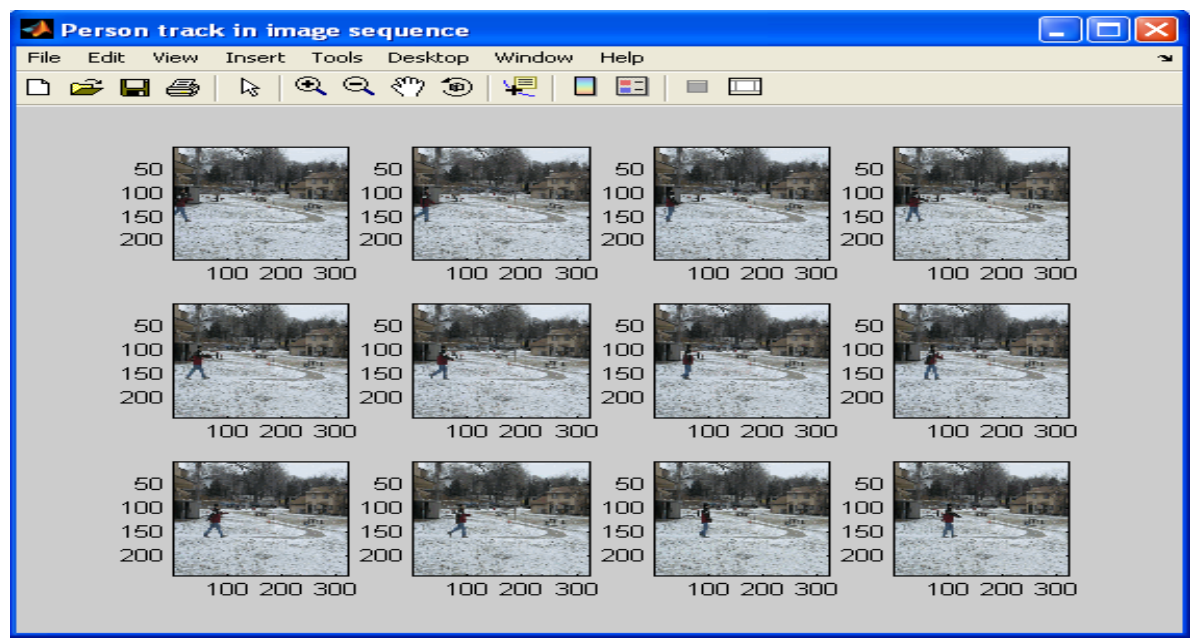

شكل (3) سلسلة الأطر للملف الفيديوي

2- اختيار المنطقة المهمة : بعد قراءة الملف الفيديوي باستخدام إيعاز (aviread) لخزن البيانات يتم عرض الإطار لإجراء المعالجة عليه باستخدام إيعاز (imshow) لاحظ الثكل (4) ويمثل

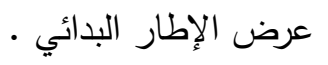

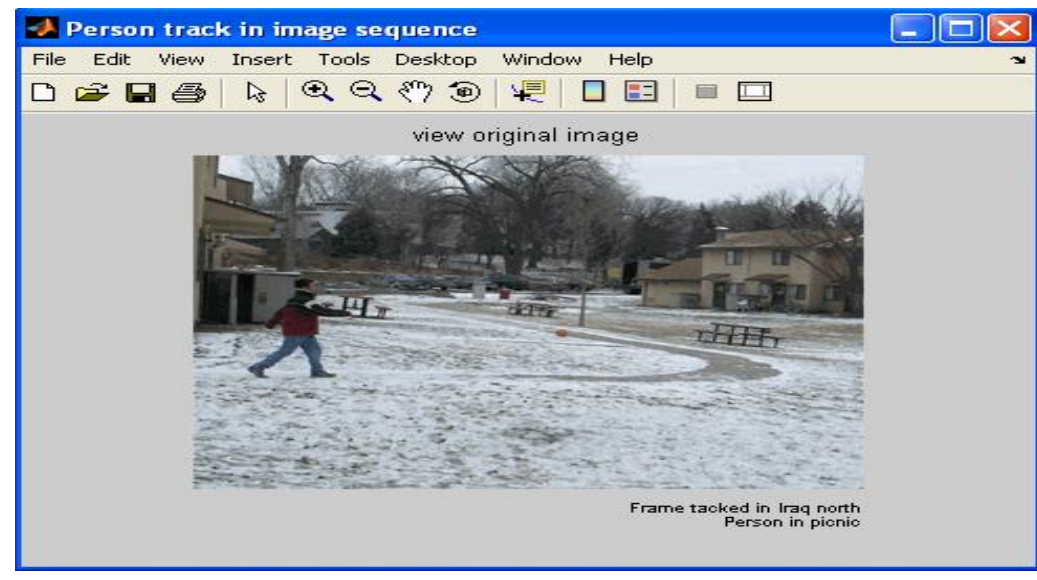

$$
\text { شكل (4) عرض الإطار البدائي }
$$

3-قص الإطار : الفائدة من عملية استقطاع جزء من الإطار لتقليل الفترة الزمنية للكثف وتمييز

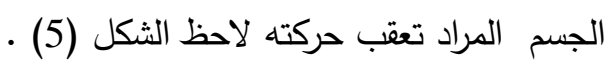




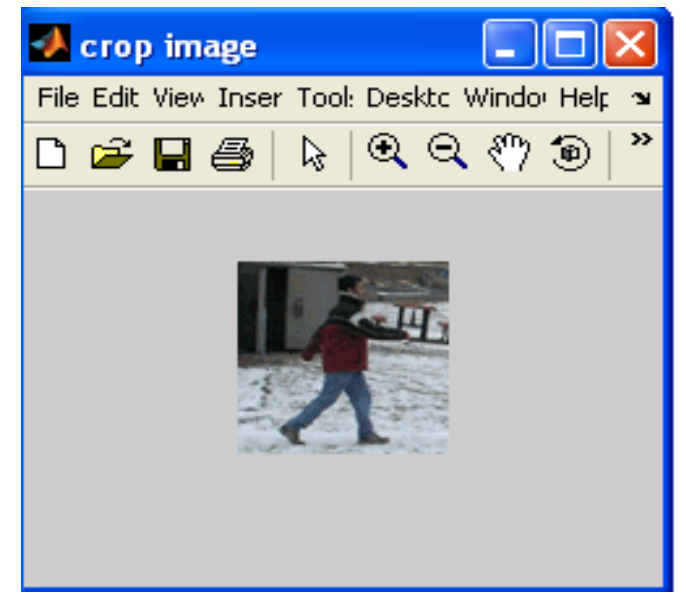

$$
\text { شكل (5) عرض جزء من الإطار }
$$

4-تحويل المنطقة المحددة إلى تدرج رمادي : يعد تحليل نسيج الصورة الرقمية من الطرائق المهمة

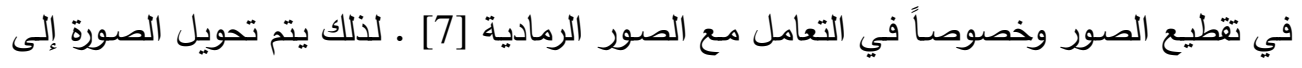

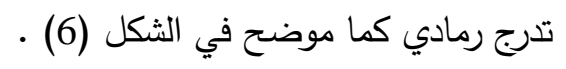

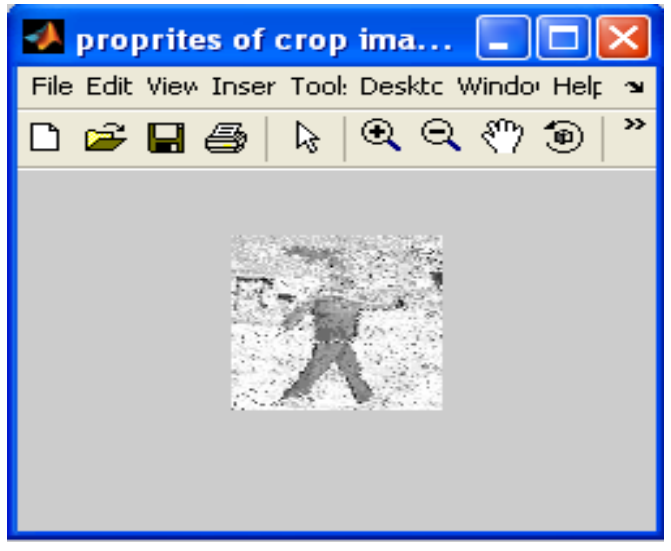

$$
\text { شكل (6) تحويل جزء الإطار إلى تدرج الرمادي }
$$

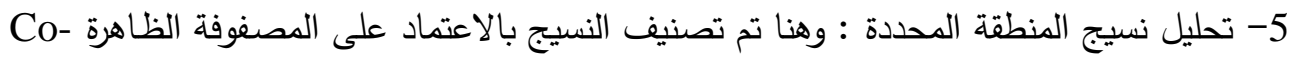

Occurrence Matrix أدناه يوضح عرض لجزء الإطار بعد تطبيق المصفوفة الظاهرة عليه . 


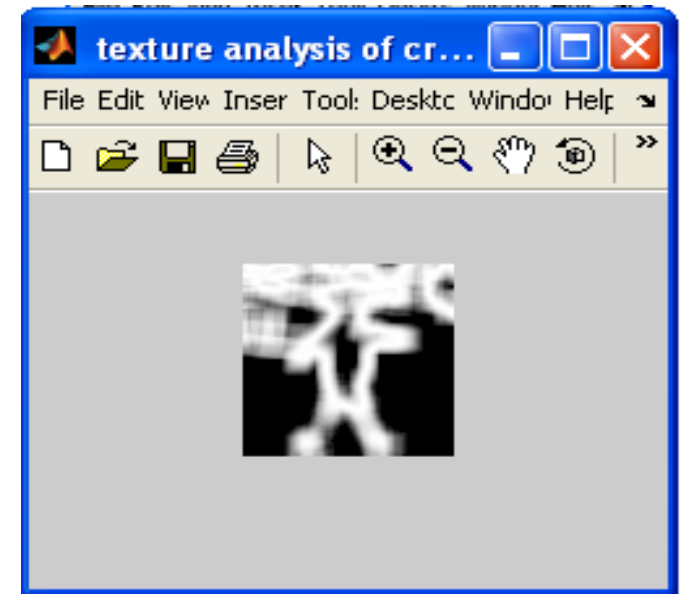

الثكل (7) تحليل جزء الإطار باستخدام المصفوفة الظاهرة

6- تمثيل الناتج باستخدام المدرج التكراري Histogram : يتم تمثيل خواص نسيج الجزء المأخوذ

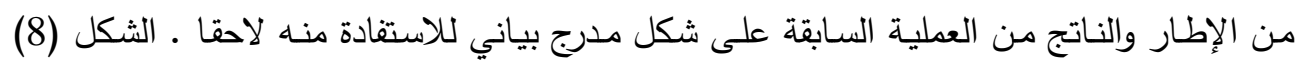
يوضح خواص نسيج لجزء الإطار .

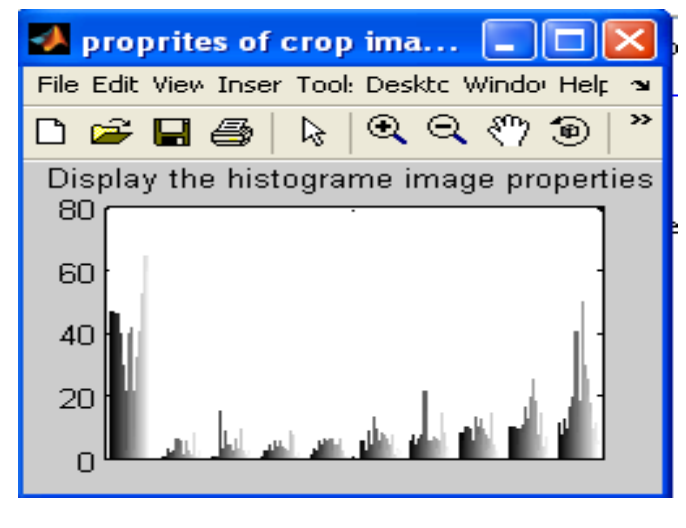

$$
\text { شكل (8) عرض خواص نسيج جزء الإطار }
$$

7- عملية تقطيع لجزء الإطار Segmentation : يتم تقطيع لجزء الإطار باستخدام التدرج البياني

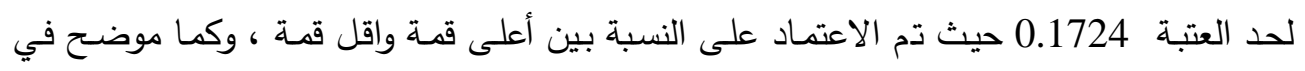

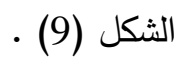




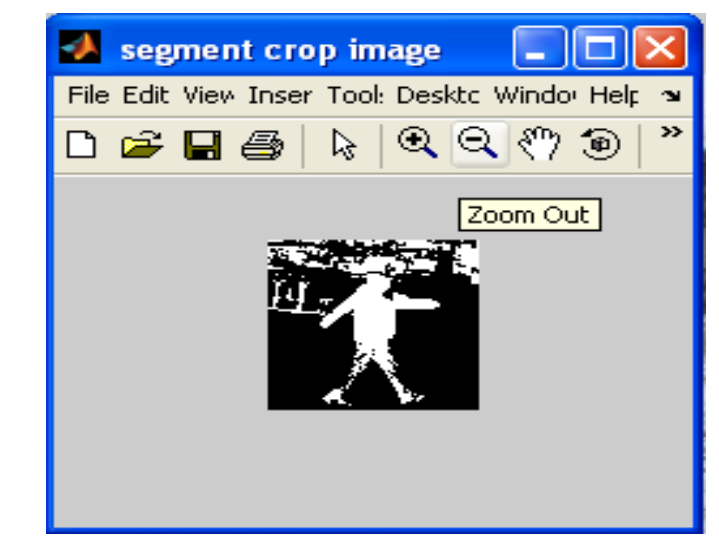

شكل (9) تقطيع الصورة باستخدام التدرج البياني لحد العتبة

8-الكثف عن الجسم : يتم الكثف عن الجسم بعد إجراء عملية تثخيص الحدود لجزء الإطار

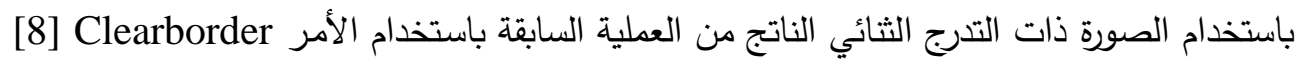
للحصول على منطقة الهدف،الثكل (10) يوضح الجسم المراد تعقب حركته.

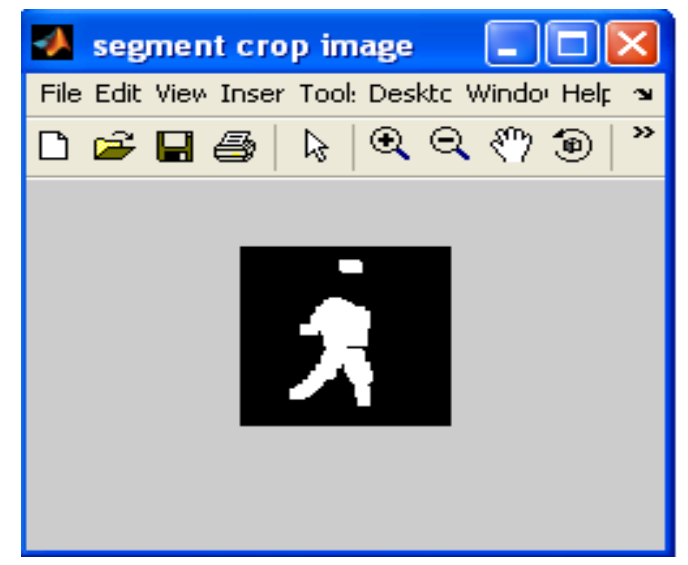

شكل (10) شكل الجسم المراد تعقب حركته

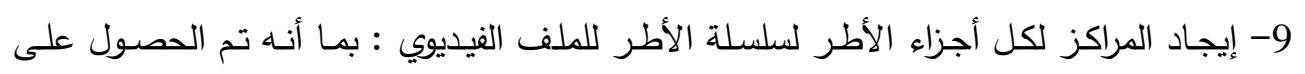

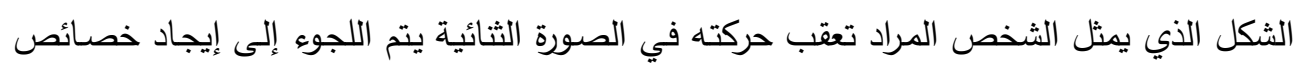

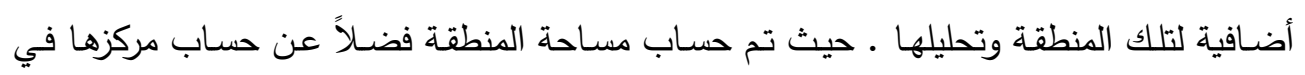
الصورة الثنائية كما في الثكل(10) المذكور سابقاً وكانت النتائج كالتالي : المساحة الكلية للمنطقة = 847 نقطة ضوئية المركز =48.5728 , 41.4733 للإحداثيتين السيني والصادي على التعاقب. 
تكرر العملية على جميع الأطر في الملف الفيديوي ويتم الاستفادة من دالة Round لتقريب الناتج

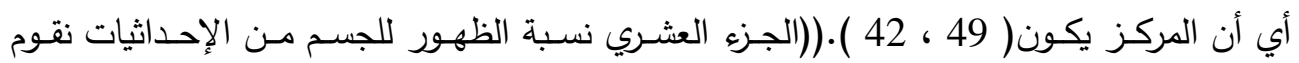
بتقريبها () 10- تعقب الجسم ويتم من خلال المسار الحركي للهدف وذلك بالاستفادة من قيم المركز التي تم

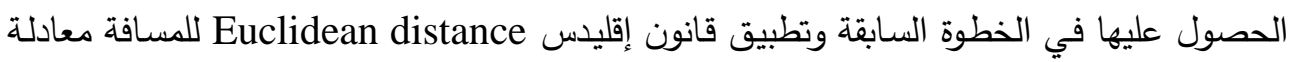
distance $\left.\left.=((\mathrm{x} 1-\mathrm{x} 2)) \cdot{ }^{\wedge} 2+(\mathrm{y} 1-\mathrm{y} 2)\right) \cdot{ }^{\wedge} 2\right) \cdot{ }^{\wedge} 0.5$ رقم (10) المذكورة سابقا.

أدناه جدول يوضح المساحة الكلية وقيمة المركز للأطر :

\begin{tabular}{|c|c|c|c|}
\hline المسافة & قيمة المركز & المساحة الكلية للمنطقة & التسلسل \\
\hline 0 & $\begin{array}{l}\mathrm{X}=40 \\
\mathrm{Y}=50\end{array}$ & 660 & .1 \\
\hline 2.3 & $\begin{array}{l}X=42 \\
Y=49\end{array}$ & 847 & .2 \\
\hline 2.3 & $\begin{array}{l}X=44 \\
Y=50\end{array}$ & 871 & .3 \\
\hline 5.2 & $\begin{array}{l}X=39 \\
Y=51\end{array}$ & 838 & .4 \\
\hline 0 & $\begin{array}{l}X=39 \\
Y=51\end{array}$ & 734 & .5 \\
\hline 6.08 & $\begin{array}{l}X=40 \\
Y=45\end{array}$ & 707 & .6 \\
\hline 2.3 & $\begin{array}{l}X=38 \\
Y=46\end{array}$ & 581 & .7 \\
\hline 1.41 & $\begin{array}{l}X=39 \\
Y=47\end{array}$ & 91 & .8 \\
\hline 1 & $\begin{array}{l}X=39 \\
Y=48\end{array}$ & 715 & .9 \\
\hline 1.41 & $\begin{array}{l}\mathrm{X}=40 \\
\mathrm{Y}=49\end{array}$ & 882 & .10 \\
\hline 1 & $\begin{array}{l}\mathrm{X}=39 \\
\mathrm{Y}=49 \\
\end{array}$ & 809 & .11 \\
\hline 12.04 & $\begin{array}{l}X=38 \\
Y=37\end{array}$ & 871 & .12 \\
\hline 14.31 & $\begin{array}{l}\mathrm{X}=41 \\
\mathrm{Y}=51\end{array}$ & 877 & .13 \\
\hline 2.3 & $\begin{array}{l}\mathrm{X}=40 \\
\mathrm{Y}=52 \\
\end{array}$ & 801 & .14 \\
\hline 2.3 & $\begin{array}{l}X=41 \\
Y=50\end{array}$ & 662 & .15 \\
\hline 6.7 & $\begin{array}{l}X=38 \\
Y=44\end{array}$ & 594 & .16 \\
\hline 7.2 & $\begin{array}{l}X=42 \\
Y=50\end{array}$ & 601 & .17 \\
\hline 2.3 & $\begin{array}{l}X=43 \\
Y=52\end{array}$ & 605 & .18 \\
\hline
\end{tabular}




\begin{tabular}{|c|c|c|c|}
\hline 3 & $\begin{array}{l}X=40 \\
Y=52\end{array}$ & 675 & .19 \\
\hline 5.38 & $\begin{array}{l}X=45 \\
Y=50\end{array}$ & 783 & .20 \\
\hline 6.708 & $\begin{array}{l}X=42 \\
Y=46\end{array}$ & 835 & .21 \\
\hline 1.41 & $\begin{array}{l}X=43 \\
Y=47\end{array}$ & 643 & .22 \\
\hline 3 & $\begin{array}{l}X=40 \\
Y=47\end{array}$ & 573 & 23 \\
\hline 5.38 & $\begin{array}{l}X=38 \\
Y=42\end{array}$ & 586 & .24 \\
\hline 6.32 & $\begin{array}{l}X=40 \\
Y=48\end{array}$ & 611 & .25 \\
\hline 3.16 & $\begin{array}{l}X=39 \\
Y=45\end{array}$ & 133 & .26 \\
\hline 1 & $\begin{array}{l}X=39 \\
Y=44\end{array}$ & 789 & .27 \\
\hline 4.47 & $\begin{array}{l}X=41 \\
Y=40\end{array}$ & 661 & .28 \\
\hline 5.2 & $\begin{array}{l}X=35 \\
Y=46\end{array}$ & 789 & .29 \\
\hline 4.12 & $\begin{array}{l}X=39 \\
Y=47\end{array}$ & 706 & .30 \\
\hline 4.47 & $\begin{array}{l}X=35 \\
Y=45\end{array}$ & 641 & .31 \\
\hline 3 & $\begin{array}{l}X=38 \\
Y=45\end{array}$ & 485 & .32 \\
\hline 1 & $\begin{array}{l}X=38 \\
Y=46\end{array}$ & 452 & .33 \\
\hline 3.60 & $\begin{array}{c}X=41 \\
Y=4\end{array}$ & 736 & .34 \\
\hline 6.4 & $\begin{array}{l}X=46 \\
Y=44\end{array}$ & 835 & .35 \\
\hline 7.07 & $\begin{array}{l}X=47 \\
Y=38\end{array}$ & 864 & .36 \\
\hline 1 & $\begin{array}{l}X=46 \\
Y=38\end{array}$ & 913 & .37 \\
\hline 1 & $\begin{array}{l}X=45 \\
Y=38\end{array}$ & 762 & .38 \\
\hline 4.47 & $\begin{array}{l}X=47 \\
Y=34\end{array}$ & 875 & .39 \\
\hline 2.24 & $\begin{array}{l}X=46 \\
Y=37\end{array}$ & 747 & .40 \\
\hline 3 & $\begin{array}{l}X=46 \\
Y=40\end{array}$ & 822 & .41 \\
\hline 5.83 & $\begin{array}{l}X=41 \\
Y=43\end{array}$ & 950 & .42 \\
\hline
\end{tabular}

11- إن نتائج تطبيق نظام المراقبة للخوارزمية المقترحة موضحة في الثكل (11) ـ 


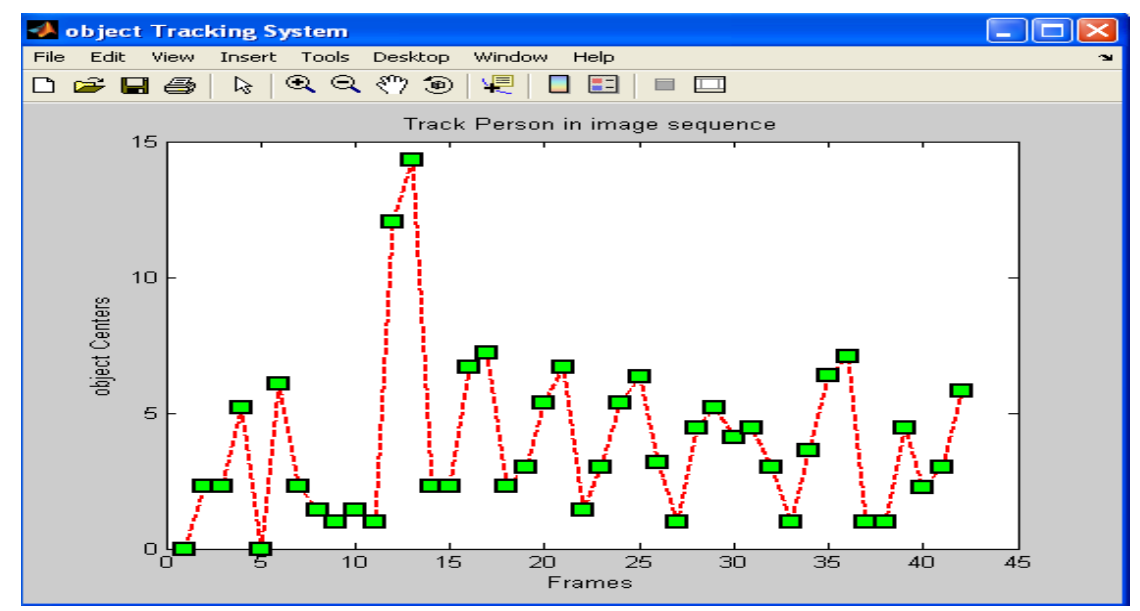

شكل (11) عرض نتائج المعالجة للخوارزمية المقترحة

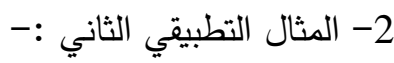

وهنا مثال أخر تم تطبيق الخوارزمية عليه يحتوي على أكثر من شخص متحرك كمـا موضح في الثكل (12) أدناه ، يتكون الملف من مجموعة من الأطر ـ نتائج المعالجة لأحد الأطر

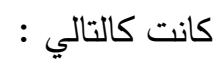
التقطيع لجزء الإطار باستخدام التدرج البياني لحد العتبة 0.1724 حيث تم الاعتماد على النسبة

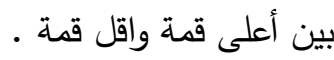
المساحة الكلية للمنطقة = 500 نقطة ضوئية للجسم بشكل مستقل . المركز =48.5728 , 41.4733 للإحداثيتين السيني والصادي على التعاقب. تكرر العملية على جميع الأطر في الملف الصوري ويتم الاستفادة من دالة Round لتقريب الناتج أي المركز يكون( 49 ، 42 ) ـ الثكل (13) يمثل عرض لسلسلة الأطر للملف الفيديوي الثكل

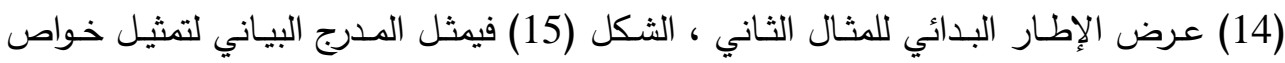
الصسورة ،أمـا الثكل (16) فيبين عملية استقطاع جزء مـن الإطـار ، والثكل (17) يبين تحويل

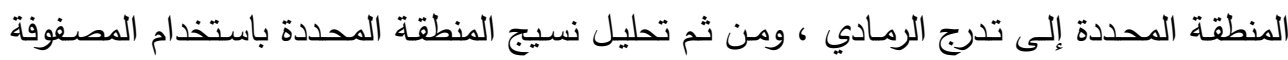

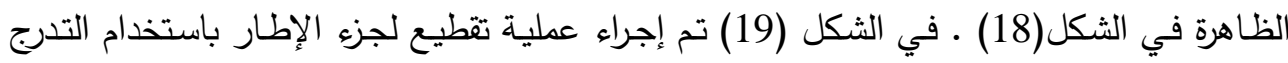
البياني لحد العتبة ، كما تم الكشف عن الأجسام الموجودة في الإطار المنتخب في الثراء الثكل (20) ، بعد ذلك في الثكل (21) تم الحصول على الثكل التقريبي للجمم المراد تعقب حركته. والثكل الثبل الأخير (22) هو عبارة عن تمثيل للمراكز التي تم الحصول عليها من تطبيق الخوارزمية والتي تمثل المسار الحركي للجسم المراد تعقبه على طول السلسلة. 


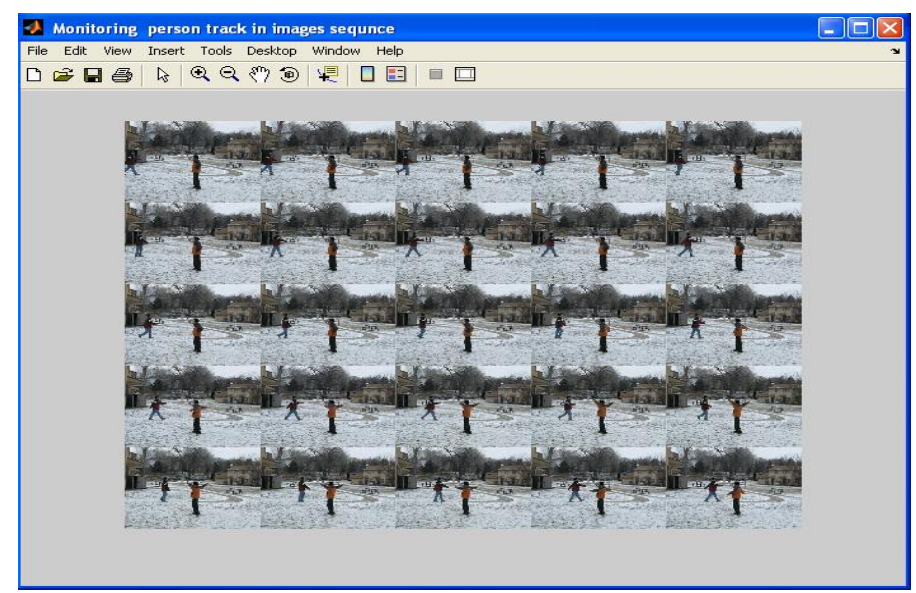

شكل (12)عرض الصور المقروء ضمن الملف الفيديوي

A Person track in images sequence

File Edit View Insert Tools Desktop Window Help

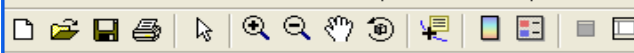
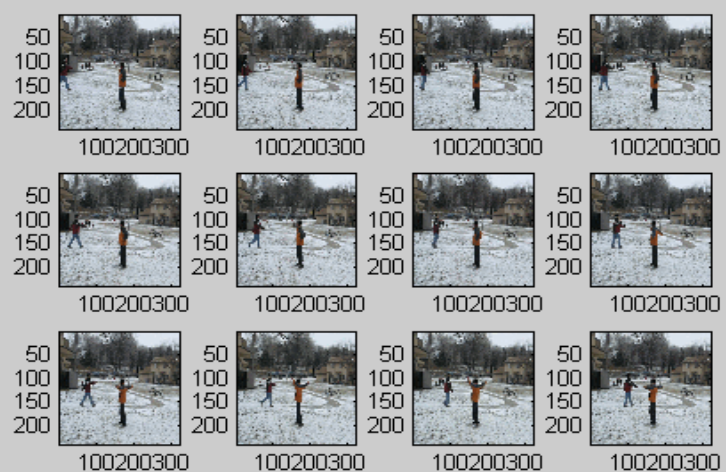

شكل (13) سلسلة الأطر للملف الفيديوي

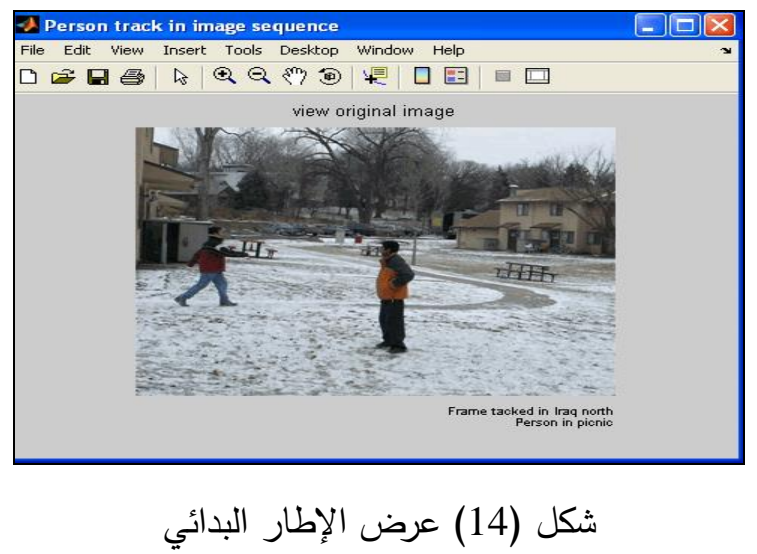




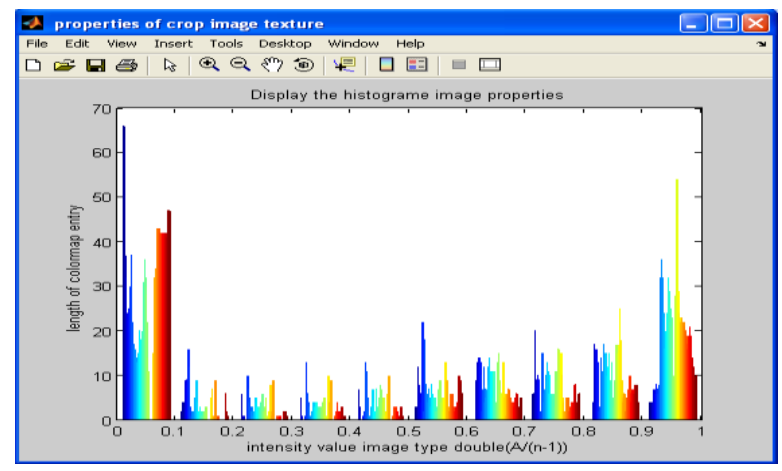

شكل (15) المدرج البياني لتمثيل خواص الصورة

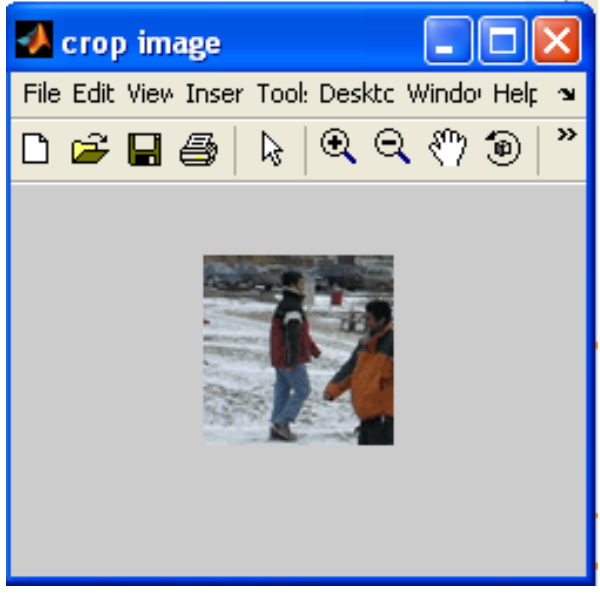

شكل(16) عرض جزء من الإطار

A crop image in gray level $\square \square x$ File Edit View Inser Tool: Desktc Windor Helf $\mathbf{v}$

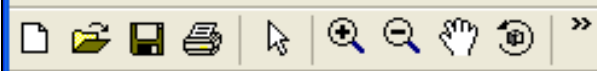

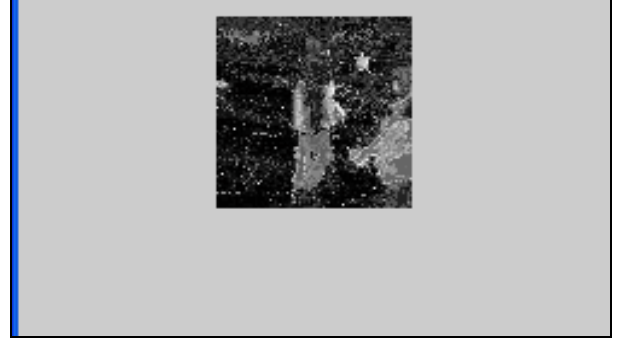

شكل (17) تحويل جزء الإطار إلى تدرج الرمادي 


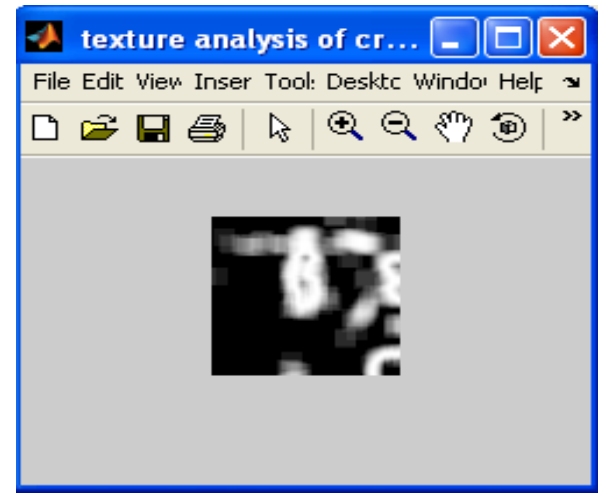

شكل (18) تحليل جزء الإطار باستخدام المصفوفة الظاهرة

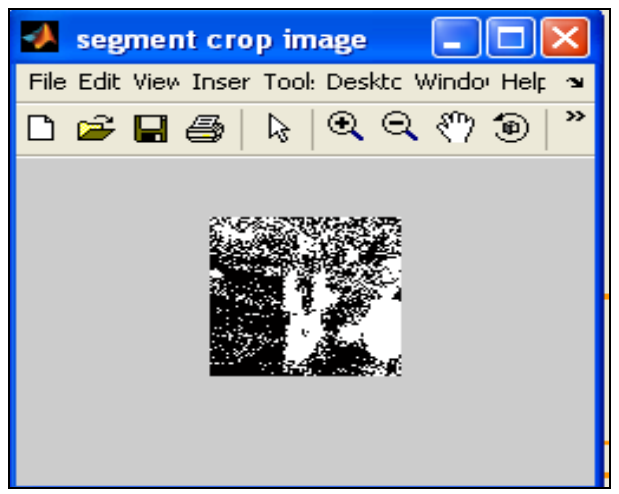

شكل (19) تقطيع الصورة باستخدام التدرج البياني لحد العتبة

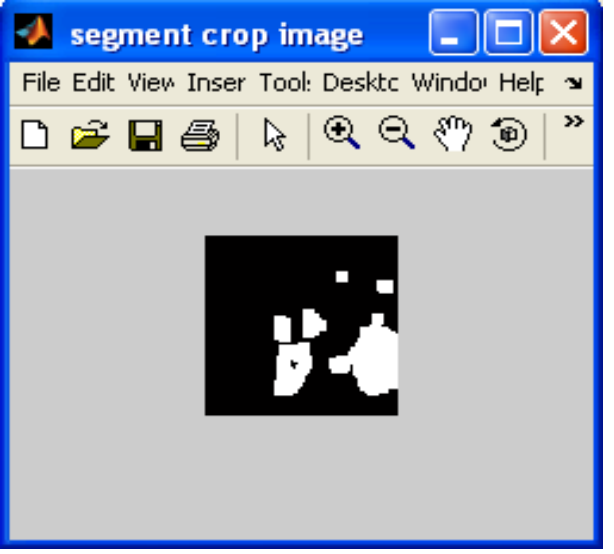

شكل (20) شكل الجسم المراد تعقب حركته مع وجود شخص أخر 


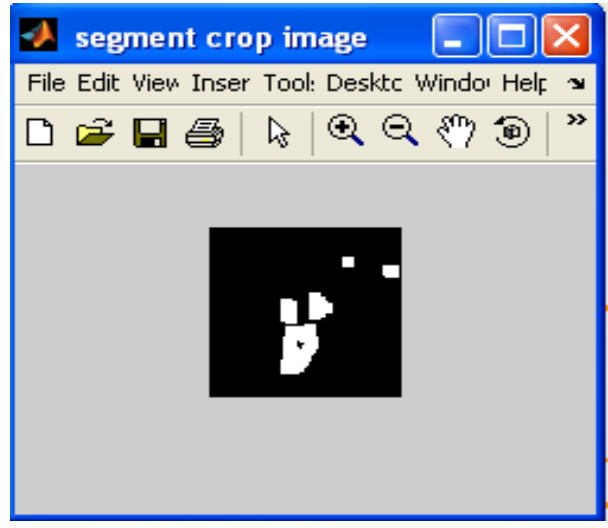

شكل (21) الثكل التقريبي للجسم المراد تعقب حركته

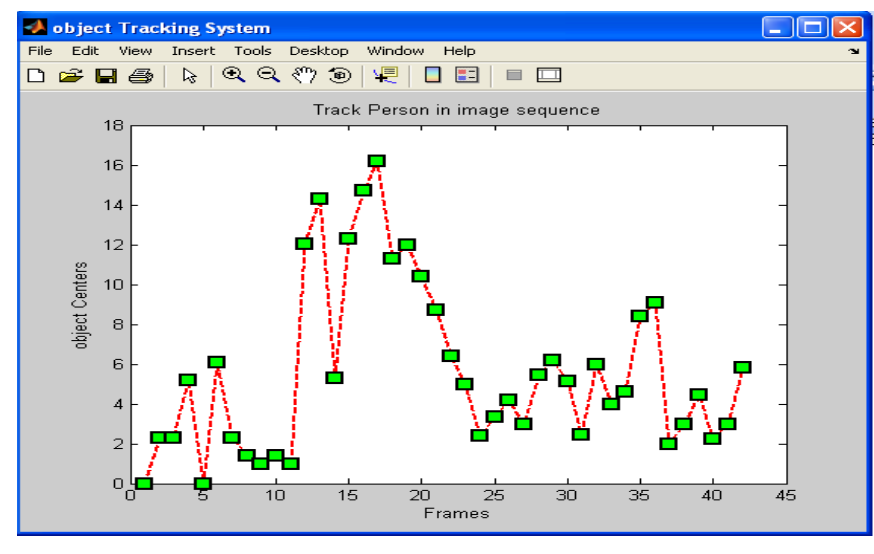

شكل (22) عرض نتائج المعالجة للخوارزمية المقترحة

4- الاستنتاجات والتوصيات:

A

من خلال تطبيق الخوارزمية المقترحة تم التوصل إلى ما يلي:

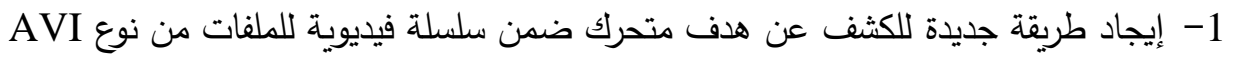

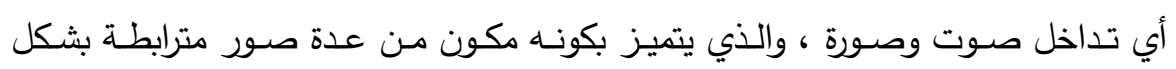
متسلسل .

2- في بعض الأحيان اللون أو الثكل أو الحركة كلا على حدى لا تكفي لعملية الكثف عن

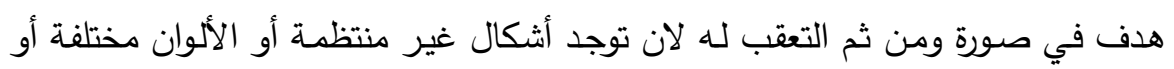
الحركة غير منتظمة لهذا تم اعتماد تقنية تحليل نسيج الصورة كطريقة مثالية لتمييز الجسم

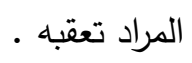


3- عملية تحليل النسيج للصورة واستخلاص خواص الصورة من أجل تقطيعها تعتبر خطوة

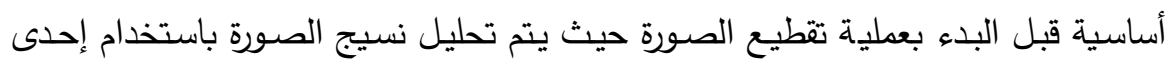

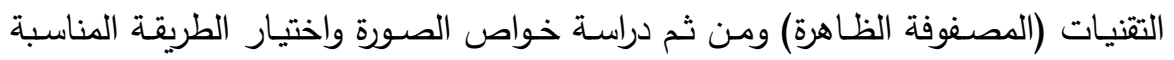

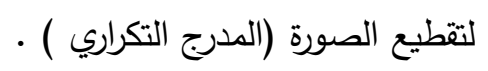

4- توجد عدة تقنيات تستخدم في تحليل نسيج الصورة لكن طريقة التحليل باستخدام المصفوفة الكرات الظاهرة اثبت كفاءتها في التطبيق المعتمد في هذا البحث ـبالإضـافة إلى اعتماد المدرج

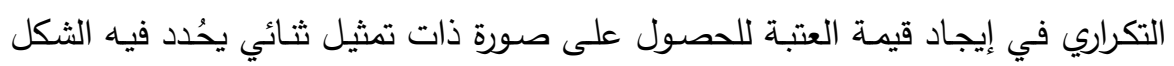
التقريبي للهذف المراد تعقب حركته .

5- تطبيق خوارزمية الكثف على طول السلسلة الصورية للملف وإيجاد مركز المنطقة طريقة

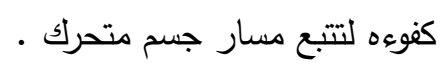

6- إن استخدام المصفوفة الظاهرة في تحليل نسيج الصورة حتى عند وجود أكثر من هدف المطلوب الكثف عنه في الأطر ذات كفاءة عالية في التطبيق المعتمد .

1- - التوصيات: الفيديوي فيه عدد الأطر المعروضـة محددة لكن ممكن تطوير العمل على ملف فيديوي يتميز باستمرارية عرض الأحداث تتم فيه حالات حركة عشوائية أو اختفاء وظهور .

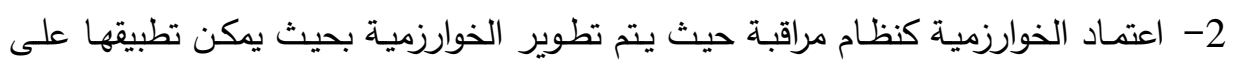
أكثر مـن جسم في وقت واحد مختلف متحرك وخاصـة في المجالات الطبيـة لإجـراء

$$
\text { التشخيصات المرضية. }
$$

3- إمكانية اعتماد الخوارزمية كنظام مراقبة لأجسام متحركة مثل مركبات وسيارات ، بهدف كثف التجاوزات وهل هناك حالات توقف أو مخالفة. 


\section{المصاد}

[1] Bautista, P. A. And Lambino, M. A. 2001, "Co-Occurrence for Wood Texture Classification", Via internet:

http://www.Physics.msuiit.edu.

[2] Gonzales, Rafael C., Woods, Richard E., (2002) "Digital Image Processing", $2^{\mathrm{ND}}$ Edition, Prentice-Hall.

[3] Guo, Zhong, (2001) "Object Detection and Tracking in Video" http://www.mcs.kent.edu/ zguo.

a.E-mail: zguo@mcs.kent.edu

[4] Leena Lepisto, Livari Kuttu, Jorma Autio And Ari Visa, 2003, "Rock Image Classification Using Non-Homogenous Textures and Spectral Imaging", Feb 3- 7, Plzen, Czech Republic. Copyright UNION Agency - Science Press.

[5] Sebe, Ismail Oner , (2002)" Object- tracking using multiple constraints" EE39j,digital video processing, winter 2002, Stanford university, EE department

iosebe@stanford.edu

www.stanford.edu/class/ee392j/Winter2002/projects/sebe_report.pdf

[6] Shanker A., Deshwal P., ( April 2002 ), "Face Detection in images: Neural networks \& Support Vector Machines".

Asim@Cse.iitk.ac.in.

Priyesd@iitk.ac.in.

[7] Toolbox, help, MATLAB 0.8

[8] Carl Philips *, Daniel Li , Jacob Furst Ph.D. , Daniela Raicu Ph.D,2008, " An Analysis of Co-Occurrence and Gabor Texture Classification in 2D and 3D "

http://facweb.cti.depaul.edu.

CPhilips@students.depaul.edu 